\title{
The quantitative motion analysis using portable gait rhythmogram after CSF drainage in the patients with idiopathic normal pressure hydrocephalus
}

\author{
Makiko Yogo ${ }^{*}$, Shusaku Omoto, Masayo Morita, Masahiko Suzuki \\ From Hydrocephalus 2015 \\ Banff, Canada. 18-21 September 2015
}

\begin{abstract}
Introduction
We previously reported that portable gait rhythmogram (PGR) equipped with accelerometers to identify gaitinduced accelerations enable us to quantitative gait analysis in Parkinson's disease (PD). We objected to reveal the quantitative gait differences between idiopathic normal pressure hydrocephalus (iNPH), PD and normal controls (NC) using PGR.
\end{abstract}

\section{Methods}

The study subjects were 7 patients with iNPH (age 76.9 \pm 2.6 years, 6 men and 1 woman), 12 patients with de novo PD who were not medicated with any kinds of anti-parkinsonian drug (age 68.3 \pm 4.5 years, 5 men and 7 women, 2 patients with Hoehn and Yahr stage I, 2 with stage II, 8 with stage III. The duration of illness was $2.3 \pm 1.6$ years.) We also studied 17 NC (age 64.7 \pm 4.5 years, 8 men and 9 women).

24 hours continuous recordings were performed twice before and after CSF drainage in iNPH patients with a trunk-mounted PGR. In PD and NC, 24hours continuous recordings were performed once at arbitrary point.

We calculated the "amount of overall movements per 24 hrs" from all motion-induced acceleration as an index of hypokinesia, "gait cycle and cadence" from the gait signals as indexes of step length, and "gait acceleration range" as an index of step power.

\section{Results}

Comparing with $\mathrm{NC}$ and $\mathrm{PD}$, the average data obtained from 6 CSF drainage responded iNPH patients showed lower "amount of overall movements per $24 \mathrm{hrs"}$. Faster "gait cycle" and higher "cadence" which indicate narrower

\footnotetext{
* Correspondence: makiko.yogo@gmail.com

Department of Neurology, The Jikei University School of Medicine, Katsushika Medical Center, Tokyo, Japan
}

step lengths, and lower "gait acceleration range" which CSF drainage were observed using PGR.

In representative case of CSF drainage responded patients, gait defects improved after CSF drainage, and the improvement lasted after LP shunt was performed. Same patients, though it was not statistical significant.

nage in an un-responded subject.

When considering percent changes of parameters, positive correlations were found between "amount of overall ( Negative correlation was found in "amount of overall movements" and $10 \mathrm{~m}$ walk time, between "gait accelera-

\section{Conclusions}

PGR revealed narrower and more monotonous step length in iNPH patients than PD and NC. And it also detected gait improvements following CSF drainage in the CSF drainage responded patients. Thus, PGR can analyze long durational gait in daily life and provide additional quantitative data on former measurements of CSF drainage easily and usefully.

Published: 18 September 2015 